\title{
HIGH PRECISION SAR FOCUSING OF TERRASAR-X EXPERIMENTAL STARING SPOTLIGHT DATA
}

\author{
Pau Prats-Iraola, Rolf Scheiber, Marc Rodríguez-Cassolà, Steffen Wollstadt, Josef Mittermayer, \\ Benjamin Bräutigam, Marco Schwerdt, Andreas Reigber and Alberto Moreira
}

\author{
German Aerospace Center (DLR), Microwaves and Radar Institute
}

\begin{abstract}
This paper addresses several innovative steps needed in the chirp scaling and extended chirp scaling (ECS) algorithms in order to process staring Spotlight TerraSAR-X (TSX) images with $21 \mathrm{~cm}$ azimuth resolution and $300 \mathrm{MHz}$ range bandwidth. The aspects that need of special consideration are the 2D phase truncation in frequency domain of ECS, the element pattern of the antenna array, the curved orbit, the stop-and-go approximation, and the troposphere. All these aspects are expounded in detail and a solution is given for each of them. The suggested corrections are applied at raw data level, hence easing the integration within the existing TSX processor. Real data acquired by TSX in the experimental staring Spotlight mode are used to validate the proposed methodology.
\end{abstract}

Index Terms - Synthetic aperture radar (SAR), Spotlight, SAR processing, troposphere

\section{INTRODUCTION}

Spaceborne SAR processing is already an established topic. Many efficient solutions have been proposed over the last decades for every mode, be it Stripmap, Spotlight, ScanSAR or TOPS. However, the assumptions of some of these algorithms start to be invalid depending on the acquisition geometry, especially in terms of the image resolution. While airborne SAR processors achieve nowadays resolutions in the decimeter range, the same approaches do not perform perfectly in a spaceborne low Earth orbit (LEO) scenario, mainly due to the greater sensor velocity and the larger distances involved. Furthermore, when getting close to decimeter resolutions several interesting effects begin to appear, which do not show up in an airborne scenario and must be taken into account to achieve a satisfactory focusing performance.

In order to design efficient processors that overcome these effects it is therefore of great help to have real data available, which in the current case are provided by the TerraSAR-X (TSX) satellite. The TerraSAR-X mission represents one of the most successful spaceborne SAR missions ever, not only because of the impressive quality and resolution of the delivered products, but also because the TSX satellite has become the perfect test-bed for future SAR missions thanks to its flexible commanding and operation. TSX has demonstrated for the first time in space several innovative modes like the TOPS (Terrain Observation by Progressive Scans) mode, spaceborne bistatic imaging (together with its twin satellite, TanDEM-X), bi-directional SAR imaging, and digital beam-forming, just to mention a few. One of the last achievements of the TSX satellite has been the acquisition of staring Spotlight (ST) data. By exploiting the

This work has been partially financed by Infoterra $\mathrm{GmbH}$, Astrium GEO-Information Services. maximum steering capability of the sensor, it is possible to achieve a steering range within \pm 2.2 degrees, hence achieving a potential azimuth resolution of $21 \mathrm{~cm}$ assuming a raised cosine window with a coefficient of 0.6 . Thanks to these data several effects could be recognized and corrected, as expounded in the following sections.

Table 1. Comparison of the Spotlight Modes. Values given for single polarization.

\begin{tabular}{ccc}
\hline Mode & Az. resolution & Az. extension \\
\hline $\mathrm{SL}$ & $1.7 \mathrm{~m}$ & $10 \mathrm{~km}$ \\
$\mathrm{HS}$ & $1.1 \mathrm{~m}$ & $5 \mathrm{~km}$ \\
$\mathrm{ST}^{*}$ & $0.21 \mathrm{~m}$ & $2.5 \mathrm{~km}-4 \mathrm{~km}$ \\
\hline${ }^{*}$ Experiment
\end{tabular}

Experimental.

\section{ST PROCESSING WITH TSX}

The operational High-Resolution (HS) and Spotlight (SL) TSX products use the so-called sliding Spotlight mode, which increases the azimuth image size of the observed spot at the expense of azimuth resolution when compared to the experimental ST mode, whose scene size is driven by the antenna footprint. Similarly, in the ST mode the beam is rotated w.r.t. the center of the scene, while in the sliding mode the rotation center is located beyond the scene [1]. Table 1 summarizes the azimuth resolution and the azimuth scene extension for the three Spotlight modes. In terms of image focusing no changes are needed in the processing kernel, but for the effects that appear due to the greater observation time, as commented next.

The operational TSX Spotlight processor uses ECS together with a sub-aperture approach to accommodate the high signal bandwidth w.r.t. the PRF, and the azimuth scaling together with SPECAN to perform the azimuth focusing [2] [3]. This algorithm performs accurately for the resolution of the operational HS mode. However, with the resolution under consideration in the ST mode, the results are not satisfactory. The detected approximations or effects are: the approximation of ECS when truncating the phase history in the 2D frequency domain; the element pattern of the antenna array; the assumption of a hyperbolic range history; the stop-and-go approximation; and the troposphere. All these aspects are analysed in detail in the following sections.

The solutions proposed in the following are oriented to minimze the effort of a potential operationalization of the ST mode. Therefore, the ECS kernel is kept and, as it turns out, it is sufficient to apply all the corrections at raw data level. It is important to mention that, due to the relative small range bandwidth $(300 \mathrm{MHz})$ and small swath (about $10 \mathrm{~km}$ ), other ECS approximations like the assumption of a range-independent effective velocity during RCMC and a bulk secondary range compression (SRC) are still valid. 


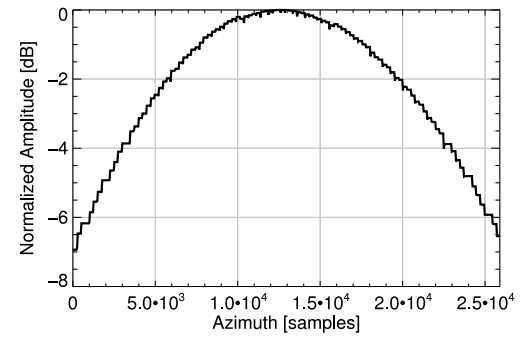

Fig. 1. Amplitude modulation introduced by the antenna array element when steering the TSX antenna within $\pm 2.2^{\circ}$.

\subsection{ECS Approximation}

The ECS algorithm truncates the square-root of the SAR signal phase in the 2D frequency domain into a Taylor series up to second order neglecting higher order terms. Due to the larger observation time of the staring Spotlight acquisition, the residual error cannot be neglected anymore. A sufficient solution in the present case is to perform a bulk correction for mid-range of the residual error, which would be performed in the $2 \mathrm{D}$ frequency domain at the raw data level. To avoid modifying the ECS kernel, only the differential phase is corrected, i.e. the difference between the square-root and the quadratic approximation. The equations can be found e.g. in [4].

\subsection{Element Pattern of Antenna Array}

Due to the large steering angles w.r.t. the size of the antenna array element, a significant amplitude modulation given by the single element antenna pattern will result during the acquisition. Fig. 1 shows the computed modulation using the TSX antenna patterns for a given ST data take. The inverse of this curve can be applied directly at raw data level to correct for the undesired modulation, similar as recently proposed in [5] for the scalloping correction of TOPS data. Note also that the inter-element distance of the antenna array ( $0.4 \mathrm{~m}$ for TSX) plays an important role, since, together with the antenna element pattern, results in the so-called grating lobes. Precisely with the maximum steering of $\pm 2.2^{\circ}$ the grating lobe has exactly the same gain as the main lobe. Both the array element pattern and the grating lobes have an important impact in terms of azimuth ambiguities and must be precisely considered for the commanding of the experimental ST mode (see [1] for details).

\subsection{The Curved Orbit}

A hyperbolic range history is assumed by most spaceborne SAR processors, but this approximation becomes less accurate the larger the observation time. For an observation time of about 7 seconds, as it is the case in the TSX ST mode, this approximation introduces a cubic-like error that degrades the azimuth resolution and introduces asymmetric sidelobes (see Fig. 2). Some solutions exist in the literature that use a numerical approach to cope with this problem, e.g. [6]. In the current solution also a numerical approach has been selected, which resembles the motion compensation approach in airborne systems [7]. The line-of-sight (LOS) approximation error shown in Fig. 2, which has been computed for a reference target in the middle of the scene, can be used to shift the data in range, both in terms of envelope and phase. With such a step, a pure hyperbolic phase history is forced, so that a conventional hyperbolic kernel can still be used to process the data without modifications. This approach assumes that the signal is monochromatic, but this is not critical due

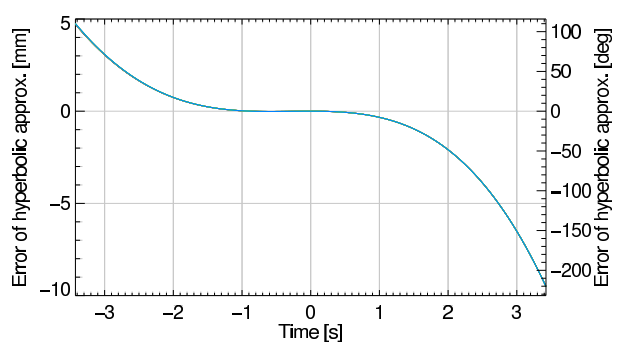

Fig. 2. Error of the hyperbolic approximation using an effective velocity. The duration corresponds to a ST data take.

to the small relative bandwidth in the TSX case. On the other hand, it has the advantage that it can cope with the azimuth variance of the geometry. Effectively, the impulse response function is azimuthvariant due to the curved orbit and the earth rotation. A correction of the above approximation error in the range-Doppler domain would introduce defocusing for the targets shifted in azimuth away from the scene center, an effect that can be observed even for a $3 \mathrm{~km}$ scene size. For this reason the correction in azimuth-time is more appropriate in this case. Note also that this first orbit compensation (OCO) makes the beam-center approximation, i.e. the correction is only valid for the target in the middle of the beam (which is also the middle of the scene for ST), but given the small beamwidth of the TSX sensor $\left(0.33^{\circ}\right)$ the introduced error is very small. Finally, note that this correction is only valid for mid range. Therefore, a second-order OCO might be necessary, as it is usually the case with airborne systems. However, it turns out that due to the large separation between the sensor and the scene and the small swath width, the range-dependency can be neglected (the residual phase errors at edge of the bandwidth are smaller than $1^{\circ}$ ).

\subsection{Stop-and-Go Approximation}

In conventional spaceborne SAR systems the stop-and-go approximation is usually assumed, i.e., the platform does not move during the transmission and reception of the chirp signal. This approximation has two effects. A "slow-time" one, which simply implies a range-dependent azimuth shift of the focused signal, since the satellite indeed moved between the transmission and the reception (about $30 \mathrm{~m}$ in the TSX case). This can be efficiently considered with an azimuth phase ramp in the range-Doppler domain before the last azimuth FFT [3]. A second effect deals with the motion of the satellite during the transmission and reception of the chirp signal itself, i.e. a "fast-time" effect. The TSX chirp signal has a length of about $50 \mu \mathrm{s}$, which implies that the satellite has moved about $40 \mathrm{~cm}$ in the azimuth direction. One intuitive way to visualize this is by considering different azimuth phase ramps for different range frequencies, since there is a direct mapping between fast-time and range frequency. Fig. 3 shows the residual phase error in the 2D frequency domain of a corner reflector (CR) for the experimental ST mode, where the range-frequency-dependent azimuth phase ramps can be clearly visualized. The phenomenon was already discussed in [8], where the analysis was done in terms of a mis-match during the matched filtering in range, and has obviously a bigger impact in FMCW systems [9]. The needed correction to be applied in the 2D frequency domain is given by

$$
H_{\mathrm{ss}}\left(f_{a}, f_{r}\right)=\exp \left(\mathrm{j} 2 \pi f_{a} \frac{f_{r}}{K}\right), f_{r} \in\left[-\frac{f_{\mathrm{s}}}{2}, \frac{f_{\mathrm{s}}}{2}\right],
$$



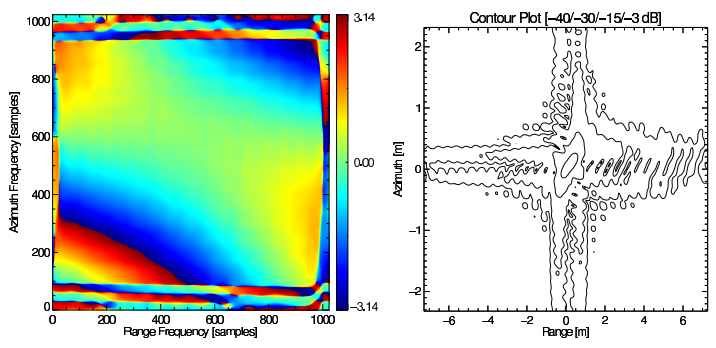

Fig. 3. The effects of the stop-and-go approximation in the experimental ST mode. (left) Residual phase of a CR in the 2D frequency domain for a ST acquisition. (right) Corresponding contour plot.

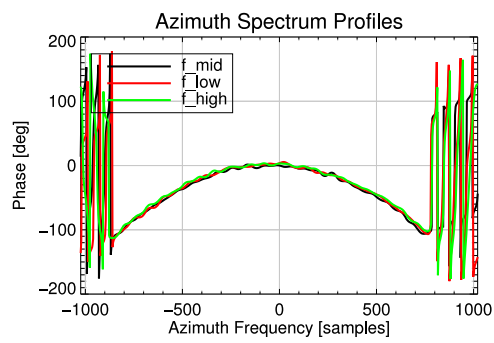

Fig. 4. Profiles along azimuth-frequency for three different range frequencies after considering the stop-and-go effect. The residual quadratic error that can be observed is due to tropospheric effects.

where $K$ is the chirp rate, $f_{r}$ the range frequency vector, and $f_{\mathrm{s}}$ is the range sampling frequency. Before the stop-and-go correction, the measured resolutions were deviating w.r.t the theoretical ones about $30 \%$ and $15 \%$ in azimuth and range, respectively. After the correction, the measured resolutions are within $14 \%$ in azimuth and $2 \%$ in range. There is still a residual quadratic phase term in azimuth as shown in Fig. 4, which has also an interesing explanation, as commented next.

\subsection{Tropospheric Effects}

Indeed, the residual quadratic phase error that can be observed in Fig. 4 is due to the troposphere. Effectively, the variation of the tropospheric delay along the synthetic aperture can be modelled as

$$
\Delta R_{\text {tropo }}\left(t ; r_{\text {ref }}\right)=\frac{Z \cdot \mathrm{e}^{-h\left(r_{\text {ref }}\right) / H}}{\cos \theta\left(r_{\text {ref }}\right) \cos \beta\left(t ; r_{\text {ref }}\right)},
$$

where $Z$ is the zenith path delay in meters, $H$ is the thickness assumed for the troposphere, $h$ is the altitude of the target, $\theta$ is the offnadir angle, and $\beta$ is the azimuth angle during the synthetic aperture. In the ST mode the dependence with $\beta$ is not negligible, hence introducing defocusing and phase errors if not considered. Furthermore, the delay in absolute terms, which in the present case shifts the image about 6 pixels in the slant-range dimension, implies a mis-match of the azimuth compression filter, introducing additional defocusing and phase errors. Using the system parameters of the present data take one gets a value of about $50^{\circ}$ due to the troposphere and another further $50^{\circ}$ due to the azimuth filter mis-match at the edge of the processed bandwidth, resulting in the total $100^{\circ}$ that can be observed in Fig. 4. Note that they are not cancelling each other, but adding with the same sign. The correction of the troposphere using (2) can be applied together with the first OCO, since, again, a bulk

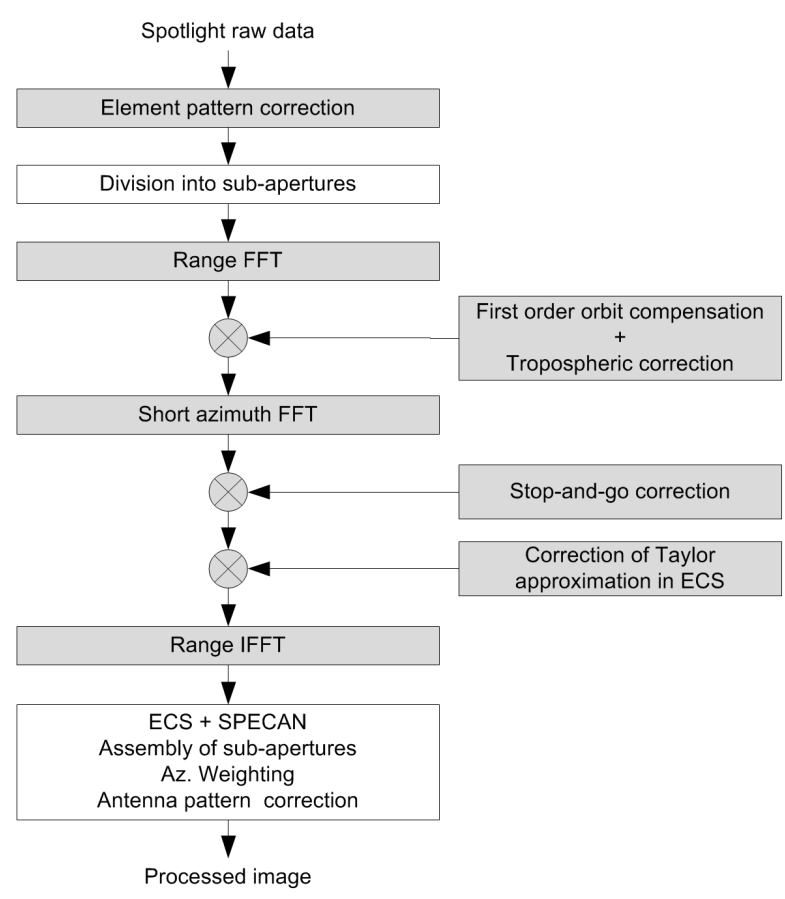

Fig. 5. Proposed update of the ECS algorithm (new steps in gray) to process data acquired in the experimental ST mode.

correction for the middle of the scene suffices.

\subsection{Proposed Processing Flow}

Fig. 5 shows the suggested processing chain for the experimental ST mode, which includes the corrections mentioned in the previous sections. First, the element pattern correction is performed at raw data level. After the division into sub-apertures and a range FFT, the first OCO and the tropospheric correction are applied together through an azimuth-dependent phase function to correct both the phase and the envelope of the signal. After going to the 2D frequency domain, the stop-and-go correction defined in (1) and the residual correction of the ECS approximation are applied. An inverse range FFT brings the signal to the range-Doppler domain, where the ECS algorithm proceeds as usual. Note that the proposed methodology can be applied with any other processing kernel, e.g. $\omega$-k or range-Doppler. In such cases, the residual ECS correction is obviously not needed.

\section{EXPERIMENTAL RESULTS WITH TSX}

Real data acquired by TSX in the experimental staring Spotlight mode have been used to validate the proposed methodology. Fig. 6 shows the impulse response analysis of two CRs located in a scene acquired in the experimental ST mode over Oberpfaffenhofen, Germany, where all the anomalies were considered and corrected. Without spectral weighting, the measured values are $44.9 \mathrm{~cm}$ and $16.4 \mathrm{~cm}$ in range and azimuth, respectively, hence matching the theoretical ones with $2 \%$ accuracy. The reflectivity image where the analysed CRs appear can be seen in [1]. Fig. 7 shows a zoom of it and compares it with the operational HS mode. Both have been multilooked to a resolution of $1 \mathrm{~m} \times 1 \mathrm{~m}$, and the radiometric resolution improvement of the ST image is evident. Note also that 

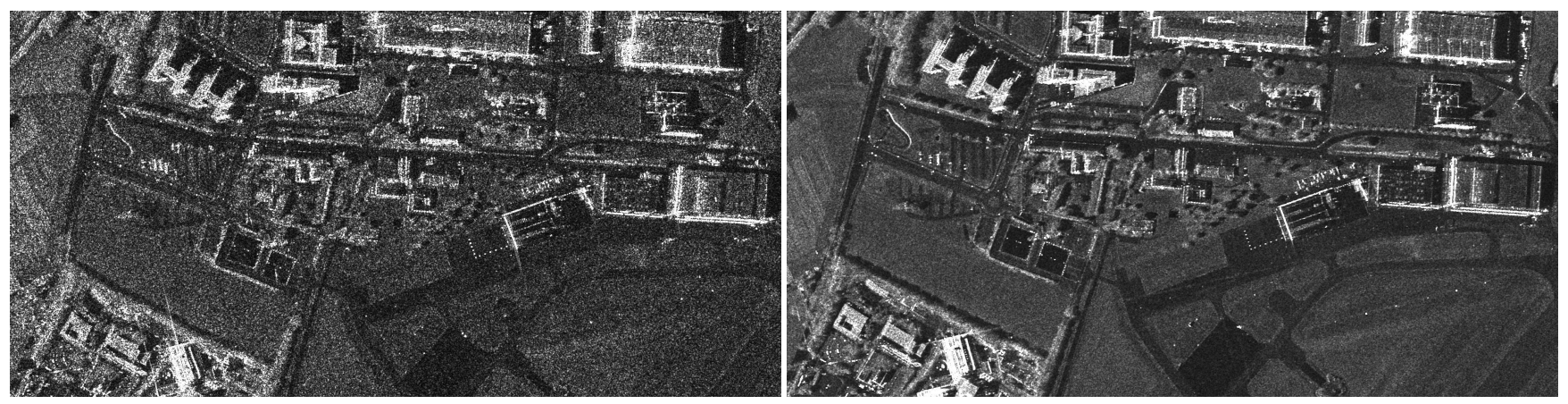

Fig. 7. Geocoded zooms over Oberpfaffenhofen, Germany, for the scene shown in [1] for the (left) operational HS mode and the (right) experimental ST mode. The resolution of the multilooked images in both cases is approximately $1 \mathrm{~m}$. Zoom size: $1000 \mathrm{~m} \times 500 \mathrm{~m}$.
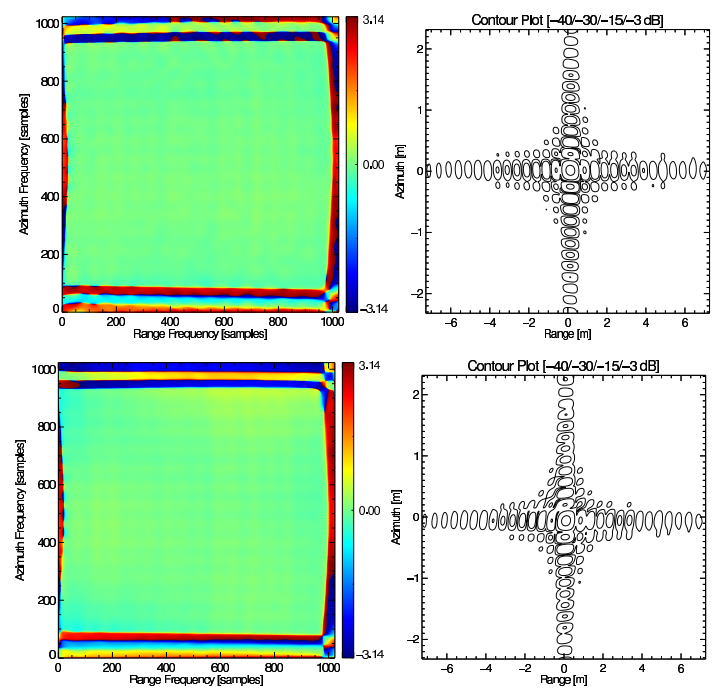

Fig. 6. Impulse response analysis of a corner reflector in the TSX scene after correcting all the anomalies. (left) Residual phase in the 2D frequency domain and (right) contour plot. The plots at the bottom row correspond to a CR located at the corner of the scene.

there is a gain in the detectability of point targets (about $7 \mathrm{~dB}$ ) in the full resolution ST data due to the increased compression gain.

\section{CONCLUSION}

This paper has presented several innovative aspects for the processing of high resolution spaceborne data, of which the stop-and-go approximation and the troposphere play an important role. Real data acquired by TSX with an experimental ST mode have been used for demonstration purposes, achieving an azimuth resolution of $16 \mathrm{~cm}$ without spectral weighting $(21 \mathrm{~cm}$ with the usual TSX weighting function). A detailed performance analysis of the experimental ST mode can be found in a parallel contribution in [1]. Note that the final performance of a future TSX ST operational product might vary w.r.t. the values mentioned in this paper.

\section{REFERENCES}

[1] J. Mittermayer, S. Wollstadt, P. Prats, R. Scheiber, and W. Koppe, "Staring spotlight imaging with TerraSAR-X," in Proc. IEEE International Geoscience and Remote Sensing Symposium (IGARSS'12), Munich, Germany, July 22-27, 2012.

[2] J. Mittermayer, R. Lord, and E. Boerner, "Sliding spotlight SAR processing for TerraSAR-X using a new formulation of the extended chirp scaling algorithm," in Proc. IEEE International Geoscience and Remote Sensing Symposium (IGARSS'03), Toulouse, France, July 21-25, 2003, vol. 3, pp. 1462-1464.

[3] Helko Breit, Thomas Fritz, Ulrich Balss, Marie Lachaise, Andreas Niedermeier, and Martin Vonavka, "TerraSAR-X SAR processing and products," IEEE Trans. Geosci. Remote Sens., vol. 48, no. 2, pp. 727-740, Feb. 2010.

[4] I. G. Cumming and F. H. Wong, Digital Processing of Synthetic Aperture Radar Data. Algorithms and Implementation, Artech House, Boston, London, 2005.

[5] Steffen Wollstadt, Pau Prats, Markus Bachmann, Josef Mittermayer, and Rolf Scheiber, "Scalloping correction in TOPS imaging mode SAR data," IEEE Geosci. Remote Sens. Lett., vol. 9, no. 4, July 2012.

[6] Davide D'Aria and Andrea Monti Guarnieri, "High-resolution spaceborne SAR focusing by SVD-Stolt," IEEE Geosci. Remote Sens. Lett., vol. 4, no. 4, pp. 639-643, Oct. 2007.

[7] A. Moreira, J. Mittermayer, and R. Scheiber, "Extended chirp scaling algorithm for air- and spaceborne SAR data processing in stripmap and ScanSAR imaging modes," IEEE Trans. Geosci. Remote Sens., vol. 34, no. 5, pp. 1123-1136, Sept. 1996.

[8] J. C. Curlander and R. N. McDonough, Synthetic Aperture Radar: Systems and Signal Processing, John Wiley \& Sons, New York, USA, 1991.

[9] Adriano Meta, Peter Hoogeboom, and Leo P. Lighthart, "Signal processing for FMCW SAR," IEEE Trans. Geosci. Remote Sens., vol. 45, no. 11, pp. 3519-3532, Nov. 2007. 